\title{
The Re-Emergence of Percutaneous Fasciotomy in the Management of Dupuytren's Disease
}

\author{
James Donaldson* and Nicholas Goddard
}

Department of Trauma and Orthopaedics, Royal Free Hospital, Hampstead, London, UK

\begin{abstract}
Dupuytren's disease is a common condition. Its management has gradually evolved but still remains a source of much controversy. Recently there has been a resurgence in the popularity of percutaneous needle fasciotomy. It is a simple method that uses a hypodermic needle as a scalpel blade. It is usually performed in the out-patient setting under local anaesthesia without a tourniquet. It has few complications and allows almost immediate return to work with few restrictions.

It can provide complete deformity correction and may offer a long-term solution in selected patients. It is also useful in converting advanced contractures into milder deformities, allowing a second stage digito-palmar fasciectomy to be more successful. Recurrence is earlier than with more formal and invasive techniques but the procedure can be repeated and does not preclude the patient from further surgery.

This article reviews the technique and assesses the efficacy and outcomes of published data.
\end{abstract}

Keywords: Dupuytren's disease, percutaneous needle fasciotomy.

\section{INTRODUCTION}

Dupuytren's disease is a chronic fibrosing disorder consisting of pathologic production and deposition of collagen in the palmar fascia of the hand. Consequently nodules and cords develop causing a potentially debilitating flexion contracture of the metacarpophalangeal (MCP) joint, proximal interphalangeal (PIP) joint and even the distal interphalangeal (DIP) joint. Dupuytren's contracture is most commonly observed in persons of Northern European descent and affects $4-6 \%$ of Caucasians worldwide [1]. The ring finger is most commonly involved, followed by the little finger and then the middle finger. The index finger and the thumb are typically spared.

The cause of Dupuytren's disease is largely unknown but a family history is often present. Males are three times as likely to develop the disease and are more likely to have a more severe form [2]. Male predominance may be related to expression of androgen receptors in the palmar fascia [3]. Additional risk factors include family history, anti-epileptics, diabetes and hyperlipidaemia.

Dupuytren's disease is common and historically has been surrounded by much controversy. Indeed its origins in the medical literature have been widely debated. The disease bears its name after the French surgeon, Baron Guillaume Dupuytren, who meticulously presented the clinical and anatomic features in 1831. Prior to that an Englishman, Henry Cline in 1777, and his successor, Astley Cooper in 1822, had previously described, published and treated the contractures. It seems, however, they were all beaten to it by Felix Plater, of Basel in Switzerland, who in 1614 described

*Address correspondence to this author at the Department of Trauma and Orthopaedics, Royal Free Hospital, Hampstead, London, UK;

Tel: 02077940500; Fax: 02077940501;

E-mail: jamesrdonaldson@gmail.com the condition in a mason with flexion contractures of his ring and little fingers, and identified the palmar aponeurosis as the source of pathology [4].

Traditional management has involved observation, fasciotomy, limited fasciectomy, total fasciectomy and dermo-fasciectomy. Like most surgical techniques, these have evolved and although recurrence rates and complications have been reduced, their occurrence is not insignificant. This has shifted the emphasis towards less invasive techniques and, in particular, percutaneous needle fasciotomy.

Percutaneous needle fasciotomy is not a new technique and was first performed with a bistoury (a long narrow bladed knife) in England by Henry Cline in 1787. It was reintroduced on the continent in the early 1970s and popularised by the French rheumatologists Lermusiaux and Debeyre [5], and later Badois [6]. In the UK it has only recently gained popularity.

\section{TECHNIQUE}

\section{Indications and Principles}

The procedure aims to divide the diseased cord by the use of the bevel of the needle and thereby restore extension of the PIP and MCP joints. Best results are seen in early disease and with more proximal (MCP joint) involvement. A palpable cord must be present with adequate overlying skin. Where full correction is unlikely to be achieved with needle fasciotomy (for example in combined MCP and PIP joint contractures), it remains a useful first stage procedure allowing the skin to stretch out before a formal second stage digito-palmar fasciectomy.

It is usually performed in the out-patient setting without the use of a tourniquet. Contraindications include infiltrating disease, inaccessible multiple cords, post-surgical digital 
recurrences, rapid recurrences in young patients (Dupuytren's diathesis), and severe digital disease causing PIP joint stiffness.

\section{Surgical Anatomy}

Portals are best planned in areas where the skin is soft, non-adherent and where the cord is a discrete linear structure. If more than one portal is needed, they are spaced at least $5 \mathrm{~mm}$ apart. Fasciotomies at the palmar level are safe - the neurovascular structures run deep to the subcutaneous fat. Distal to the MCP joint care is needed as the anatomy becomes distorted and risk of neurovascular injury is higher. Only midline and prevascular cords, which are extended under the skin, are accessible.

To avoid tendon damage the patient is intermittently asked to actively flex and extend the PIP and DIP joints to demonstrate the presence or absence of needle motion with active tendon excursion. If the needle moves with the finger it suggests inadvertent entry into the flexor tendon sheath and the needle should be re-positioned. The procedure and post-operative protocol are explained to the patient, including the importance of reporting any finger paraesthesia or numbness and avoiding any sudden movements.

\section{Preparation}

The patient washes their hands with soap and water. The skin is then cleaned with alcoholic disinfectant and 1-3mls of $1 \%$ lignocaine are injected using a 29-31 gauge hypodermic needle and syringe - an insulin syringe is particularly useful. Sterile drapes and gloves are not required. The skin and subdermal tissue around the Dupuytren cord of interest are infiltrated. It is important to keep the needle in the subdermal layer to avoid neurovascular and tendon injury. Cords are insensate (neurovascular structures and flexor tendon sheaths are not) and this allows inadvertent injury to be avoided. Some experts in fact do not use local anaesthetic, as they believe it may obscure inadvertent digital nerve damage. The patient should be sitting or recumbent to avoid potential vasovagal complications.

\section{Procedure}

The aim is to provide a transverse fasciotomy deep to the skin where the cord is a discrete linear structure. Nodules and skin creases should be avoided. Skin creases are generally in close proximity to the flexor tendon sheath and are also potential sites for skin tears. Nodules have little effect on the deformity or its correction. A 23 gauge (blue) hypodermic needle is inserted through the skin and the dermis. The tip of the needle is placed under the skin perpendicular to the cord. A reciprocating motion is used to define the extent and surface geometry of the cord. There are different ways to divide the cord once the anatomy has been defined. The senior author prefers a 'sawing' type action with the needle at ninety degrees to the cord and using the bevel of the needle on the surface and dividing it incrementally from superficial to deep. Another option is the multiple stab technique, which some authors believe to be safer - in that the patient may experience sensory changes if the digital nerve is encountered.
The cords must be held under tension to allow the needle to 'cut' and to pull the cord up and away from the deeper structures. A sensation of resistance should be felt and the fascia should feel crisp when cut - this is often audible. Passive stretching may be done after each release, which pulls the ends of the cord apart and obtains maximal release of the contracture. The process may be repeated if necessary, from proximal to distal, if there is still cord causing a residual contracture. For advanced Dupuytren's successive sessions can be proposed. Some authors advocate the use of Doppler examination where there is a possibility of a spiral cord in order to avoid neurovascular injury [7].

In addition some authors advocate the use of corticosteroid injections at the time of fasciotomy or soon after to soften the cord plus or minus any co-existing nodules. There is little evidence to suggest performing this routinely and is not the senior author's preferred technique.

\section{Post-Procedure}

A simple dressing can be applied to the wound and the patient can continue with their normal activities. Physical therapy is not usually needed but the patient is advised to regularly stretch out their finger. Occasionally a splint may be beneficial. If there has been a tear in the skin, strenuous gripping or heavy manual labour should be avoided until it has healed - similar to the McCash technique.

\section{Complications}

Skin tears may occur which may need bandaging until they heal. They are more common in and adjacent to flexion creases. Other complications include nerve injury and flexor tendon injury, which are both rare.

\section{Clinical Example}

A 56 year old right hand dominant male with progressive Dupuytren's contracture seen in the out-patients department for the first time. His deformity largely involves the MCP joint of the little finger. It currently restricting his work as a mechanic.

Local anaesthetic is injected after consideration of appropriate portal placement. A blue hypodermic needle is inserted and the bevel and tip are used to divide the cord. The patient is monitored continually for fingertip numbness (digital nerve injury or contusion) and needle movement with active tendon excursion (insertion of the needle into the flexor tendon). Complete correction is achieved. The patient will be followed up serially to assess for recurrence and if necessary the procedure can be repeated.

If the cord feels rubbery or is no longer being divided the needle can be changed for a fresh one. The patient is educated on the likelihood of recurrence (as with all Dupuytren's procedures) and post-operative stretching (see Fig. 1a-e).

\section{DISCUSSION}

There is a huge amount of literature regarding the treatment of Dupuytren's disease, but few quality studies assessing one technique over another. Van Rijssen et al., [8] performed a prospective randomised controlled trial 
(a)

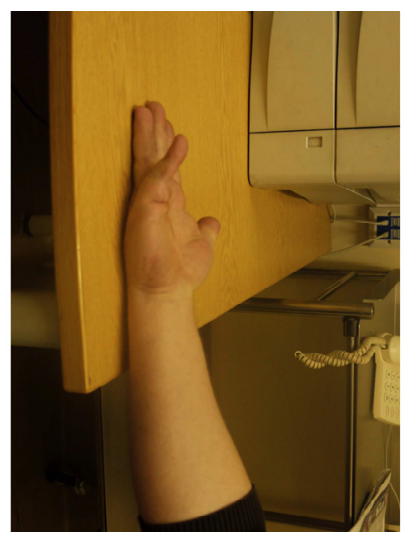

(b)

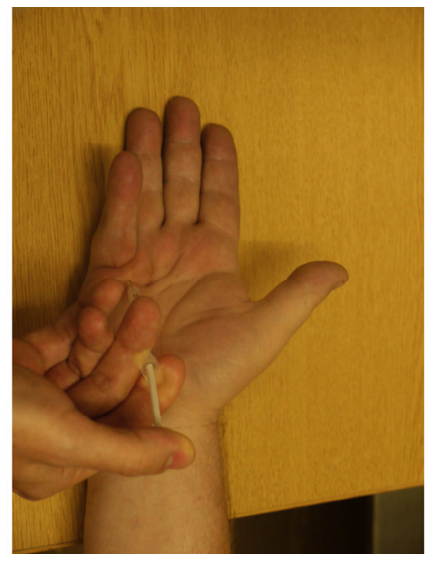

(c)
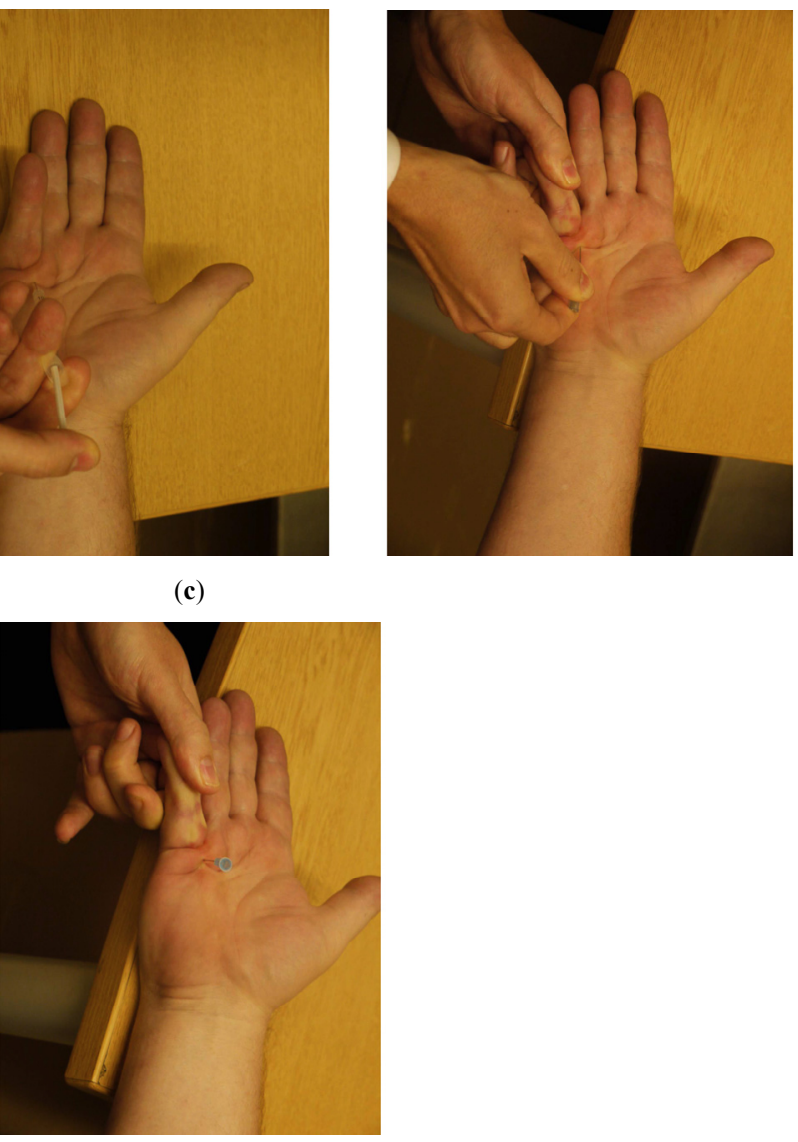

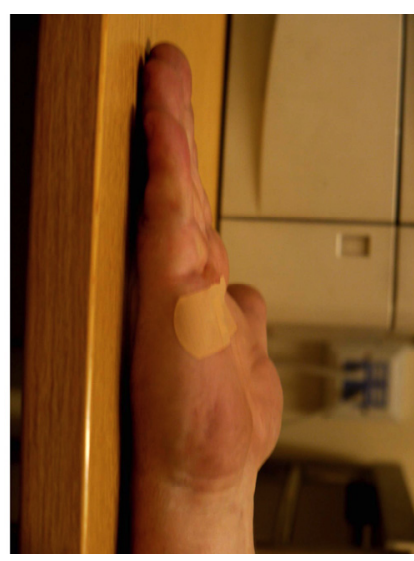

(d)

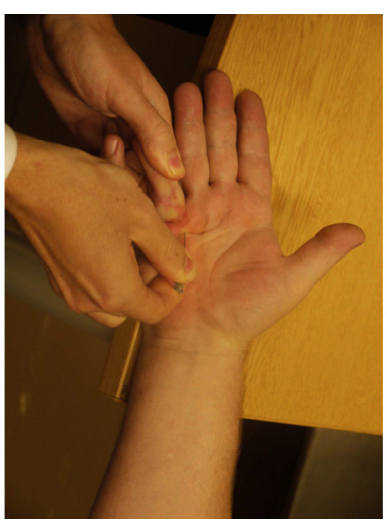

(e)

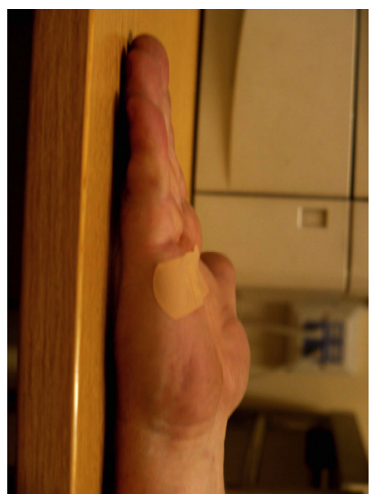

Fig. (1). a) The pre-procedure deformity; b) injection of local anaesthetic; c) needle placement; d) needle fasciotomy with tension on the finger; e) post-procedure result with complete deformity correction. 
comparing percutaneous needle fasciotomy and limited open fasciectomy in 117 hands. For Tubiana stages 1 and 2 (see Table 1) percutaneous fasciotomy was equal to limited fasciectomy in efficacy. For Tubiana stage 3 and 4 disease limited fasciectomy was superior. Limited fasciectomy was, however, associated with a 5\% major complication rate (compared to zero major complications in the needle fasciotomy group). In addition pain scores and satisfaction with hand function were consistently better in the percutaneous fasciotomy group. The authors did find a higher proportion of minor complications in the needle fasciotomy group (38\% compared to $17 \%)$ but all of these resolved with no intervention.

Table 1. Tubiana Classification of Dupuytren Contracture [9, 10]

\begin{tabular}{|c|l|}
\hline Stage $\mathbf{1}$ & $\begin{array}{l}\text { Combined flexion contracture of MCP and PIP joints less } \\
\text { than } 45 \text { degrees }\end{array}$ \\
\hline Stage 2 & Combined flexion contracture between 45 and 90 degrees \\
\hline Stage 3 & Between 90 and 135 degrees \\
\hline Stage 4 & Greater than 135 degrees \\
\hline
\end{tabular}

Badois et al., [6] reported on a multi-centre study involving 952 hands in 799 patients. A total of 3736 needle fasciotomies were performed. An improvement in more than $70 \%$ was achieved in $92.6 \%$ of stage 1 cases; in $77.7 \%$ of stage 2 cases; in $71.2 \%$ of stage 3 cases and in $56.6 \%$ of stage 4. Their complications were two ruptured flexor tendons, two nerve sections, skin cracks in $2 \%$ and temporary dysaesthesia in $0.8 \%$.

A further study by Lermusiaux [11] involved digital involvement only. Thirty-two patients and 42 fingers were prospectively included for percutaneous needle fasciotomy. Three months after treatment the rate of good results was $79 \%$. A skin fissure was noted in one but no other adverse events were reported. They concluded the technique is safe and suitable for palmar and digital involvement.

A further case series assessed 90 patients and 123 hands for five years after one to three percutaneous fasciotomy sessions [6]. In total $66 \%$ of patients were followed up. The mean Tubiana score improved from 3.15 to 0.66 . The mean rate of good results was $81 \%$ : $92 \%$ for Tubiana stage $1 ; 89 \%$ for stage $2 ; 83 \%$ for stage 3 and $48 \%$ for stage 4 . At five years the mean Tubiana score remained low, at 0.99 ; the mean rate of good results was $69 \%$ (92\% for stage $1 ; 74 \%$ for stage $2 ; 57 \%$ for stage 3 and $3 \%$ for stage 4 ). Five year recurrence was $50 \%$ for all cases. Adverse events included skin fissures in $16 \%$ and transient dysaesthesia in $2 \%$. The authors noted greatest improvements were seen in the MCP joints (79-100\% improvement), whereas PIP joints improve, but to a lesser extent (46-76\%).

Three case series reported on short-term structural efficacy on needle fasciotomy [12-14]. Immediate or 1 week post treatment assessments showed 76-77\% global improvement. There was $79-100 \%$ improvement for MCP joints, $46-76 \%$ for PIP joints and 75\% for DIP joints.
The most common complications are skin fissures and transient nerve paraesthesias. Both generally settle down with little future consequence. Skin fissures may need regular dressings, especially if they are full thickness, and strenuous activities should be avoided until they heal. Permanent nerve injury and flexor tendon ruptures have been reported but are rare: Tubiana and Thomine [15] $-0.2 \%$; Foucher [16] - 0.05\%.

Recurrence is always a problem with any Dupuytren's treatment and will inevitably occur over time. Van Rijssen et al., reported a $65 \%$ recurrence rate at average follow-up of 32 months [14]. Bleton [17] reviewed 59 fingers with a 73 degree average pre-operative deficit. He noted after 6 months that $85 \%$ of the patients did not have any MCP joint deficit, but $51 \%$ had a recurrent PIP deficit.

Percutaneous needle fasciotomy has several advantages over open techniques:

- It has lower, less serious, complication rates.

- Most patients recover rapidly and there are few, if any, restrictions after the procedure.

- $\quad$ The procedure is performed in the out-patient setting, under local anaesthesia.

- It has a similar short-term outcome and higher initial satisfaction among patients [8] compared to open procedures, especially in the case of milder contractures, or with contractures largely involving the MCP joint.

- In some patients it may represent a long-term solution.

- $\quad$ There is a significant cost saving compared to formal surgical procedures [18].

It is not, however, a substitute for fasciectomy and formal surgical procedures in all cases but a useful tool in the reconstructive 'ladder of treatment' for Dupuytren's disease.

\section{CONCLUSION}

Percutaneous needle fasciotomy has been shown to be beneficial in Dupuytren's disease and the short-term structural efficacy is well documented. It is a simple and quick method, with a short sick leave period for the patient, limited care requirements and a low overall cost. Recurrence rates are higher but major complications are avoided and the procedure can be repeated multiple times. It does not prohibit other treatments and may take a patient from a Tubiana stage 3 or 4 to a stage 2, allowing a more successful second stage digito-palmar fasciectomy.

Better results are seen at earlier stages and with more proximal disease. It must, however, be practised by experienced clinicians with an understanding of the anatomy and patho-anatomy of Dupuytren's contractures to minimise any potential complications.

\section{ACKNOWLEDGEMENT}

None declared.

\section{CONFLICT OF INTEREST}

None declared. 


\section{REFERENCES}

[1] Hurst LC, Badalamente MA, Hentz VR, et al. Injectable collagenase clostridium histolyticum for Dupuytren's contracture. N Engl J Med 2009; 361(10): 968-79.

[2] Anthony SG, Lozano-Calderon SA, Simmons BP, Jupiter JB. Gender ratio of Dupuytren's disease in the modern U.S. population. Hand (NY) 2008; 3(2): 87-90.

[3] Al-Qattan MM. Factors in the pathogenesis of Dupuytren's contracture. J Hand Surg Am 2006; 31(9): 1527-34.

[4] Belusa L, Selzer AM, Partecke BD. Description of Dupuytren disease by the Basel physician and anatomist Felix Plater in 1614. Handchir Mikrochir Plast Chir 1995; 27(5): 272-5.

[5] Lermusiaux JL. Le traitement medical de la maladie de Dupuytren. In : de Seze S, Ryckewaert A, Kahn MF, Guerin CL, Eds. L'actualité rhumatologique. Paris: Expansion Scientifique Francise 1979; pp. 338-43.

[6] Badois FJ, Lermusiaux JL, Massé C, Kuntz D. Non-surgical treatment of Dupuytren disease using needle fasciotomy. Rev Rhum Ed Fr 1993; 60(11): 808-13.

[7] Short WH, Watson HK. Prediction of the spiral nerve in Dupuytren's contracture. J Hand Surg Am 1982; 7(1): 84-6.

[8] van Rijssen AL, Gerbrandy FS, Ter Linden H, Klip H, Werker PM. A comparison of the direct outcomes of percutaneous needle fasciotomy and limited fasciectomy for Dupuytren's disease: a 6week follow-up study. J Hand Surg Am 2006; 31(5): 717-25.
[9]

Tubiana R, Michon J, Thomine JM. Scheme for the assessment of deformities in Dupuytren's disease. Surg Clin North Am 1968; 48(5): 979-84.

[10] Tubiana, R, Michon J, Thomine JM. Assessment of deformity in Dupuytren's disease. In: Hueston JT, Tubiana R, Eds. Dupuytren's Disease. $3^{\text {rd }}$ ed. Philadelphia USA: Churchill Livingstone 1974.

[11] Lermusiaux JL, Badois F, Lellouche H. Dupuytren's Disease. Rev Rhum (Ed Fr) 2001; 68: 542-7.

[12] Foucher G, Medina J, Navarro R. Percutaneous needle aponeurotomy: complications and results. J Hand Surg $\operatorname{Br} 2003$; 28(5): 427-31.

[13] Cheng HS, Hung LK, Tse WL, Ho PC. Needle aponeurotomy for Dupuytren's contracture. J Orthop Surg Hong Kong 2008; 16(1): 88-90.

[14] van Rijssen AL, Werker PM. Percutaneous needle fasciotomy in dupuytren's disease. J Hand Surg Br 2006; 31(5): 498-501.

[15] Tubiana, R, Thomine JM, Brown S. Complications in surgery of Dupuytren's contracture. Plast Reconstr Surg 1967; 39(6): 603-12.

[16] Foucher, Medina GJ, Malizos K. Percutaneous needle fasciotomy in dupuytren disease. Tech Hand Up Extrem Surg 2001; 5(3): 161 4.

[17] Saffar AP, Foucher G, Bleton R, Marcireau D, Alnot JY. Treatment of Dupuytren's disease by percutaneous needle fasciotomy. In: Saffor P, Amadio PC, Faucher G, Eds. Current Practice in Hand Surgery. London: Martin Duritz 1997; pp. 187-93.

[18] Webb JA, Stothard J. Cost minimisation using clinic-based treatment for common hand conditions--a prospective economic analysis. Ann R Coll Surg Engl 2009; 91(2): 135-9.

(C) Donaldson and Goddard; Licensee Bentham Open.

This is an open access article licensed under the terms of the Creative Commons Attribution Non-Commercial License (http://creativecommons.org/licenses/by-nc/3.0/) which permits unrestricted, non-commercial use, distribution and reproduction in any medium, provided the work is properly cited. 